\title{
What is the Future of Therapeutic Education? The Italian Scenario and the Covid-19 Pandemic Lesson
}

\author{
M Riccio ${ }^{1 *}$, and G Memoli² \\ ${ }^{1}$ Polo Diabetologico Distrettuale, ASL Napoli 2 Nord, Distretto 38 Marano-Quarto, Italy \\ ${ }^{2}$ Centro Antidiabete San Luca, Ariano Irpino (AV), Italy
}

*Corresponding author: M Riccio, Polo Diabetologico Distrettuale, ASL Napoli 2 Nord, Distretto 38 Marano-Quarto, Italy, Tel: +39 0815768257; E-mail: michele.riccio@aslnapoli2nord.it

Received: 27 Aug, 2020 | Accepted: 08 Oct, 2020 | Published: 15 Oct, 2020

Citation: Riccio M, Memoli G (2020) What is the Future of Therapeutic Education? The Italian Scenario and the Covid-19 Pandemic Lesson. J Diab Res Ther 6(2): dx.doi.org/10.16966/2380-5544.153

Copyright: (C) 2020 Riccio M, et al. This is an open-access article distributed under the terms of the Creative Commons Attribution License, which permits unrestricted use, distribution, and reproduction in any medium, provided the original author and source are credited.

\section{Abstract}

The exponential increase in cases of Type 2 Diabetes Mellitus (T2DM), particularly in developing countries, is combined with the progressive failure of healthcare facilities to provide adequate assistance.

The role of Therapeutic Education (TE) in the treatment of T2DM has long been validated. However, despite efforts to implement educational interventions, these have proved to be largely insufficient in meeting current needs. Today, the ongoing COVID-19 pandemic requires a greater complexity of care that calls for an alternative approach in educational intervention.

Therefore, Diabetology, as well as modern Medicine, must look into digitization as a possible solution.

The increasingly widespread diffusion of internet and the means for accessing it have in fact triggered a full-fledged race to computerization, a growth that is not always consistent.

TE cannot be oblivious of these new developments. The digital world offers opportunities for reviewing and updating TE, particularly amid the COVID-19 crisis, without dismissing its recent and prestigious past.

This new TE approach however requires attention, and new skills need to be acquired and extensively tested.

Keywords: Therapeutic education; Health literacy; Digital therapeutic education; Video; Social media

\section{Keypoints}

The role of Therapeutic Education in the treatment of diabetes

The role of modern digital technologies

Why is the future digital

The fundamental aspects of Digital Therapeutic Education

\section{Background}

Both the WHO and the IDF have reported the exponential increase in the diagnosis of T2DM worldwide [1], particularly in developing countries, in parallel with changing economic conditions [2].

In Europe and Italy, however, the increase of T2DM cases has shown a slightly, less aggressive trend relative to year 2000, Italy registered in fact an increase of one million people with diabetes [3].

Along with these worrisome figures, we have been witnessing a consistent downward trend in the quality of diabetes care in Italy.

In the past few years, Italy has seen a progressive reduction both in the number of facilities for people with diabetes, $[4,5]$ and of medical and paramedic staff.
Over the last few years, recruitment and turn-over freezes, the adoption of more stringent criteria for acceptance into Medical schools, and the reduced availability of specialty courses, have in fact caused a sharp reduction in the number of physicians, in particular specialists.

According to a recent report published by ANAO/ASSOMED [6], within 6 years a shortage of about 16,500 specialists is expected, particularly Internal Medicine, Pediatrics and Endocrinology specialists who are primarily involved in the care of people with diabetes.

The progressive depletion of structural and staff resources is paired with the growing restriction of economic resources available for the treatment of Persons With Diabetes (PWD), which conflicts with the 
inevitable increase in direct and indirect costs [7]. This significantly hampers the quality of care provided to PWD $[8,9]$.

The current COVID-19 pandemic, and the application of hygienicsanitary measures for the prevention and control of the infection, have caused an increase in the complexity of care [10], forcing us to remodel our line of intervention. On the other hand, it has accelerated the process of digitization of health protocols, stressing the critical role of modern communication technologies in the treatment of chronic diseases, including diabetes mellitus [11-13].

\section{Aims of the Review}

This review aims to illustrate the outcomes and limitations of traditional therapeutic education methodologies for the PWD by comparing them with the potential of modern digital platforms, with particular attention to educational videos.

Our proposal illustrates how to improve its quality, usability and effectiveness.

\section{Methods}

We determined the archives of Pubmed, Google Scholar, Youtube and Vimeo using the following key terms: "educational therapy in diabetes" AND "mHealth" AND "educational video" AND "COVID-19 OR "2019 novel coronavirus" OR "SARS-CoV-2."

Retrieval date range was set up to April 2020.

\section{The role of Therapeutic Education (TE) in the treatment of persons with diabetes}

The crucial role of TE in the treatment of diabetes has been approved and validated by all major international LGs including the Italian ones [14-16], which have stressed its importance and indispensability in the management of PWD soon after being diagnosed with diabetes (Figure 1).

The development of TE can be traced back to 1905, when the German pathologist Karl Von Noorden began educating his diabetes patients by instructing them on nutrition with healthy cooking classes. Subsequent pioneering work, during the 20s through the 70s, was carried out by E.P. Joslin in Boston and Lawrence in London, followed by Silvestri La Penna in Rome, and by Leona Miller and Jack Goldstein in the USA [17]. In 1979, thanks to major contributions from JeanPhilippe Assal, who first theorized Therapeutic Education as we know it [18], formal guidelines took shape with the establishment of the Diabetes Education Study Group (DESG) [19].
Since then, TE has been widely seen as effective in treating chronic diseases, such as diabetes in particular [20-22].

The subsequent developments of TE had to proceed in parallel with progress in another major area of health education and promotion: Health Literacy (HL).

HL has been defined as "the cognitive and social skills which determine the motivation and ability of individuals to gain access to, understand and use information in ways which promote and maintain good health (WHO Health Promotion glossary, 1999)."

There is strong evidence that patients with low HL and diabetes benefit very little from educational interventions, even if they are well structured [23-25].

The level of HL is therefore linked to the cultural level of the people involved and, in a world as transversal as the one we live in, it also depends to a large extent on the language used to communicate, inform and educate patients. The term "language" is specifically used in its broader meaning, which spans from purely verbal to non-verbal and then to the most typical and characteristic language of our times, the multimedia language [26].

Over the last few years, several educational initiatives and projects have been launched in Italy and Europe, with the aim of not only testing the efficiency of educational tools, but also of creating and certifying their effective and functional models.

In Italy, for example, in 1996 the Department of Internal Medicine of the University of Turin launched a trial to assess the applicability and transferability of the Group Care educational model. The trial, called the Romeo Project, involved 13 Italian Diabetes Units [27,28].

In 2002, in Italy, the AMD ETS (Associazione Medici Diabetologi) (Structured Therapeutic Education) Group launched the AMD ETS Project (Figure 2), which was completed around 2009 [29]. Out of the 650 diabetes units initially recruited, only 327 took part (about 50\%) and of these only 262 implemented the courses. Only 109 of them have actually carried out the ETS courses for persons with diabetes, corresponding to less than $30 \%$ of the total number of diabetes units operating nationwide at that time [30].

In 2009, the Diabetes Conversation Educational Program [31,32] promoted by IDF Europe in cooperation with Healthy Interaction [33], which implemented the educational tool THE CONVERSATION MAPS, was launched in Italy as well.

$$
\begin{aligned}
& \text { AMD/ SID } 2018 \text { Italian standards of medical care in diabetes mellitus } \\
& \text { Patients should be educated in the self-management of diabetes at the time of diagnosis, providing for } \\
& \text { subsequent reinforcements in order to maintain the skills acquired and prevent complications, when any new factors } \\
& \text { capable of influencing self-management should occur, and at all stages of the disease. } I A \\
& \text { The educational intervention must be planned and structured. } I A \\
& \text { Education in the self-management of diabetes must be guaranteed within the team by the various } \\
& \text { professional figures (physician, nurse, dietician, socialhealth educator and other staff involved in the care setting), } \\
& \text { specifically qualified on the basis of continuous professional training in the educational activity } I A
\end{aligned}
$$

Figure 1: Levels of proof and strength of recommendations. 


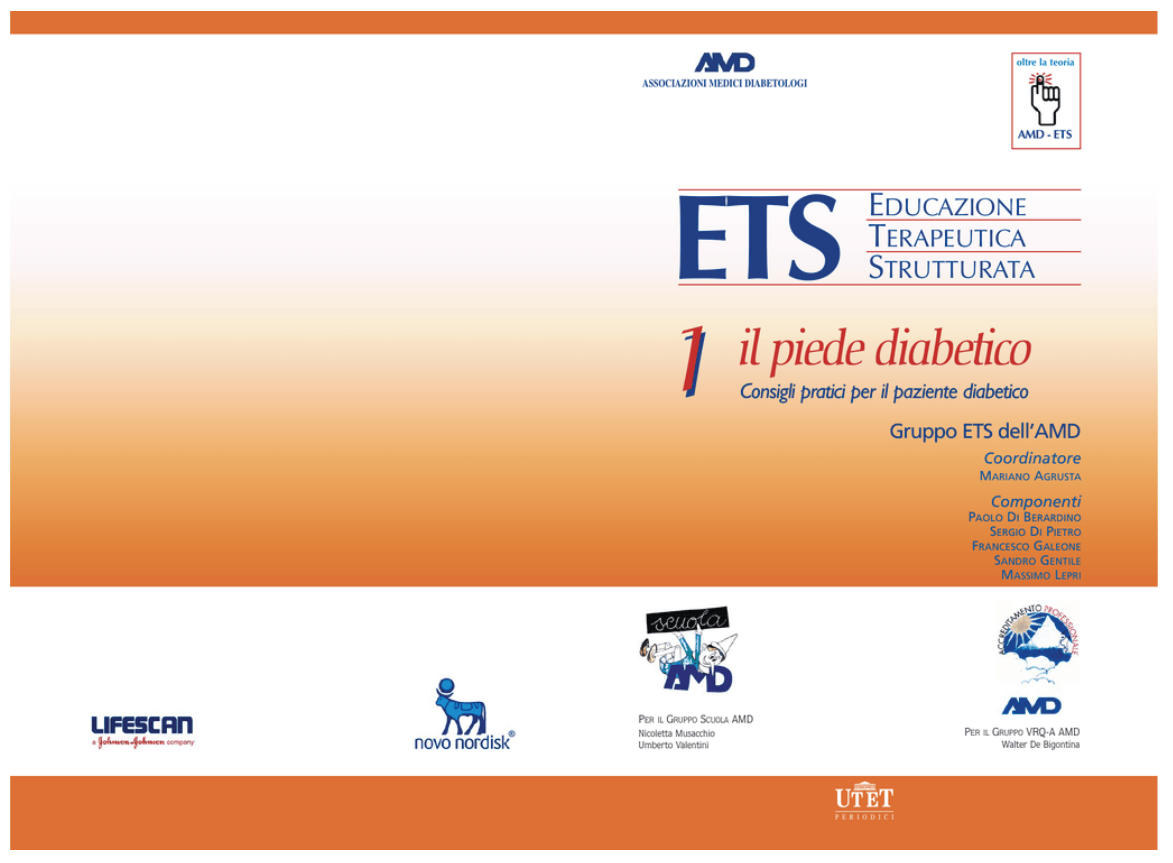

Figure 2: ETS Manual "The Diabetic Foot": Tips for people with diabetes.

It served as an interactive interface promoting conversations on the multiple aspects of diabetes among groups of patients, in which the trained health care staff acted as facilitators. The Project, coordinated by Dr M. Agrusta on behalf of GISED (Gruppo Italiano Studi sulla Educazione Terapeutica) [31], was completed in 2012 and involved 1,795 healthcare professionals and about 16,000 patients over a period of 3 years.

Once the initial enthusiasm and the impetus of institutional and non-institutional sponsors decreased, the project has slowly run out, even if it is still one of most widely used by educational teams.

All these projects yielded good results, confirming the clinical effectiveness of TE, although not all published data agree [32-37].

But all these projects highlight, once again, the limiting variables of these methodologies:

-The constant availability of health personnel, by number and specific training;

-The availability of dedicated and adequate spaces, where educational meetings can be carried out;

-The adequate recognition by the Italian National Health System (SSN) of the therapeutic accuracy of TE by giving it due time and remuneration.

\section{The role of modern digital technologies}

For some time now modern medicine, including Diabetology, has been looking for a possible workaround to restrictions posed by increasingly scarce economic and staff resources, through digitization and information technology.

These problems are even more evident and compelling in the Italian economic and social scenario.

The ongoing pandemic, which has suddenly and radically transformed the world, has once again stressed the urgent need for modern digital technologies in their full potentialities and applications, above all in the management of chronic diseases and, first and foremost, Diabetes Mellitus [38].

Hence, Telemedicine was and is an obvious and mandatory choice.

In the last decade the number of Internet accesses to health related websites, aimed at promoting changes to lifestyles and health selfmanagement has literally boomed, thanks to the opportunity to browse the Net on a 24/7 basis and the improvement of network connection technologies, through smartphones and tablets [39].

According to a report published in 2012 [40], by the Pew Research Center, $7 \%$ of smartphone users, and as much as $35 \%$ of under 35 years old users accessed health information directly from their mobile devices. The most recent Italian survey shows that about $74 \%$ of web users do health-related research.

This has caused a surge in the healthcare app market, as well as the number of internet accesses to health and self-management websites $[41,42]$.

Over the last few years, numerous publications have confirmed the effectiveness and economic efficiency of Web-based applications and interventions, for the treatment of Diabetes and other chronic diseases. Numerous systematic reviews and meta-analyses have also been dedicated to the topic [43-45].

Thus, the scientific evidence on the efficiency of the Internet in improving clinical outcomes, increasing specific knowledge, achieving behavioral and lifestyle changes and promoting participation in the management of our own health is now well established [46].

\section{Why is the future of Education Therapy Digital?}

Evidence shows that during national emergencies such as earthquakes, epidemics, and other natural disasters, the diversion of health resources and the consequent interruption of care, creates a 
strongly negative impact in the management of chronic diseases and especially diabetes [47].

This requires preparation and planning of new intervention policies aimed at mitigating the damage and filling in the gaps of conventional chronic disease management.

The advantage of a more widespread use of digital resources, including its economic benefits, is now commonly accepted by the medical scientific community, both in times of war and peace [48].

Educational proposals such as Group Care, ETS, Conversation Maps, although highly effective in pursuing and achieving their objectives, are limited, now more than ever, to a small number of elite welfare scenarios. This was made even more evident by the COVID-19 pandemic.

As a consequence, few patients have been involved, leaving behind the vast majority of PWD, particularly those in more disadvantaged contexts, where the frequency and prevalence of diabetes and its complications are higher.

It is therefore clear that choosing digitization for TE, i.e. creating the conditions for digital therapeutic education (eET), is an obvious if not necessary option. eET offers flexibility, free access, and a wide choice of content, which can be accessed anytime, anywhere.

According to the data of the Digital 2019, recently published on the Internet [49], an in-depth analysis of Italian data, allows us to appreciate that $92 \%$ of the total population browses the Web, $85 \%$ of which from mobile devices. Of these, $88 \%$ access the Web at least once a day, and $92 \%$ streams video contents.

The age group between 35 and 55 years (about 35\%) had the highest percentage of users. At the same time in Italy, the percentage of over 50 years old people accessing social media rose to around $40 \%$ [50] (Figure 3).

This implies that in the near future an overgrowing number of people with diabetes will be more familiar with network resources. These will include today's younger generations i.e. those who, with a brilliant intuition, Marc Prensky, American writer and innovator in the field of education and learning, called DIGITAL NATIVES [51] as well as older age groups, defined by Prensky as Digital Immigrants.

The choice of conveying TE through multimedia videos (Facebook groups, chats, live streaming or webinars) [13] seems therefore appealing and actually effective [52].

However, due to the risk of misinformation or diffusion of harmful health content [53], it is essential that scientific societies monitor the materials available on the web and promote verified educational initiatives [13].

The adequacy of the communication model used is an additional critical element.

The scientific and communicational quality of most videos on the Web is extremely poor, particularly from a scientific and communicational point of view. This was aptly highlighted by Gimenez-Perez G, et al. [54], who advises against the use of YouTube for providing educational support, unless ad hoc videos are made available.

However, this should not discourage us to reject from adopting this platform as a means of educational integration: as Tackett pointed out in his conclusions, "Medical education content on YouTube can immediately and consistently reach a global viewership with relevant content. Educators may consider posting videos to YouTube to reach a broad audience [55]."

On the other hand it is necessary to change the way the educational material is prepared, in order to fine-tune it to the needs and peculiarities of future people with diabetes.

We should also make sure that scientific organizations make validated and qualified material available on the Web. As pointed out by Gimenez-Perez G, et al. [54], the most qualified video material on the Web is provided by pharmaceutical companies or Health Organizations.

The COVID-19 pandemic has further highlighted these needs even more [56] and commanded an acceleration towards the use of these resources [57].

It is therefore advisable to modify the rules of health communication by adopting a language more in line with the changing times and making a more efficient and modern use of social media.

\section{The New Era of Digital Therapeutic Education (eET)}

To create an effective eET, two fundamental aspects need to be addressed:

- The choice of a medium through which educational content can be offered

- The way in which the multimedia contents should be prepared

The correct application of these key points is essential for the success of the educational intervention, as they guarantee the greatest dissemination, satisfaction and engagement of patients.

\section{Choosing the Medium}

During the Covid-19 pandemic it has become even more apparent how the use of social media can effectively contribute to educational interventions [56,57].

There are two possible scenarios which do not exclude, and actually complement, each other.

In the first scenario, the educational intervention is provided similarly to traditional options, i.e. through webinars and/or live streaming, web conference etc and make use of digital platforms such as Zoom, Skype, Google Meet, Microsoft Teams, etc. [13].

It should be facilitated and managed by an official health organization, and by appropriately trained health-care personnel.

In fact, if managed by untrained health personnel or supported by organizations that have exclusive commercial interests, these intervention can lead to the dissemination of misleading and even harmful information [55].

Further limitation includes the lack of that empathic environment which makes educational counseling effective and constructive.

The second option is to provide educational videos by conveying them through the different social platforms such as YouTube, Instagram, TikTok, etc. They have the potential to act as an important vehicle for sharing and disseminating important health information. They can also be a means through which users can interact and socialize through the creation of blogs and forums $[58,59]$.

These videos should be produced, offered and managed by recognized professional health organizations, the only ones able to guarantee the accuracy and quality of health information. 


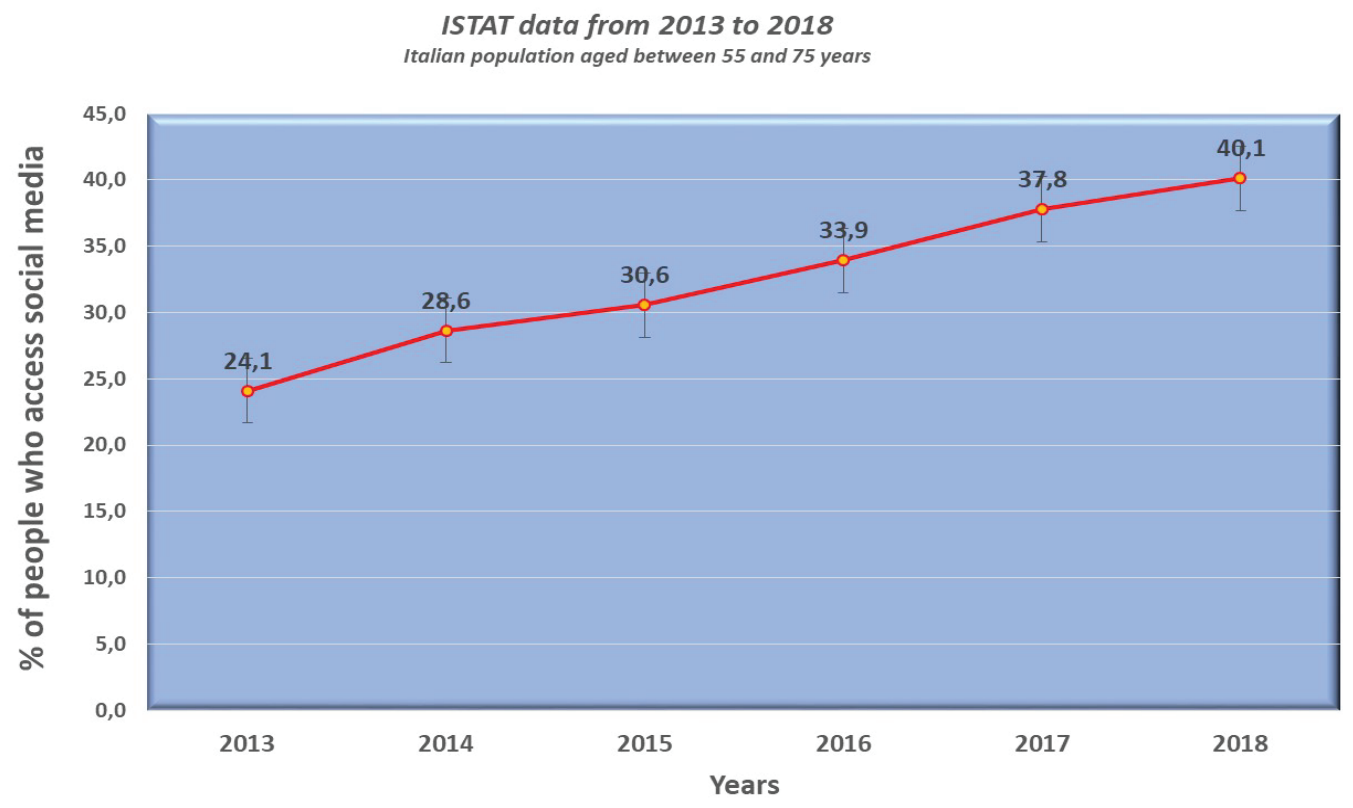

Figure 3: Percentage of Italians aged 55-75 - access to social media - from 2013 to 2018.

As Gabarron E, et al. [60] reports, young people currently prefer videos as a source of health information. No similar data are currently available for people over 60 .

Nevertheless, many of the videos hosted on social media, not only contain incorrect, contradictory, and misleading information [5358], but are also ineffective from communicational point of view, as they are built in a crafty and inadequate way. Even some of the videos developed by professional and governmental organizations, while scientifically accurate, present these limitations.

The possibility of accessing the educational material on a 24/7 basis, choosing when, how and where is the right moment to view them, is among the main advantages offered by social platforms.

In addition, social media not only make it possible for us to easily reach our patients, but also allow us to monitor their level of satisfaction, through "likes" and comments, giving us the opportunity to better target our interventions, and adjusting them to the interests and needs of users.

We should also keep in mind the possibility of using this material in the "FOCUS Groups" spontaneously created within social networks, where each user can freely discuss and confront with others.

As Tenderich highlighted [59] "Social media have become crucial for patient empowerment and peer support. This support transcends mental health concerns to actually provide patients with important practical tips and tricks for diabetes self-care and product selection/ decision-making information from peers. In conclusion, he says "Social media provides a significant source not only of moral support and camaraderie, but also critical education on thriving with diabetes. Importantly, we observed strong evidence of peer influence on patients' therapy and diabetes technology purchasing decisions".

\section{Create Novel Educational Video Standards}

For some time now the most common digital platforms (YouTube ${ }^{\mathrm{mm}}$ and/or Vimeo $^{\text {tix }}$ ) have been presenting numerous examples of Educational Videos (EV) [53]. However EVs available on the Web, are often times limited in terms of technical quality, scientific value, communicative effectiveness and educational and dissemination capacity [61].

Hence scientific organizations should strictly monitor the production and dissemination of scientific information and this is certainly not an easy task.

One possible solution is that Health Public Organizations produce validated and certified EV materials, focusing on new communicational and educational video standards which would match the characteristics of both current and future users, meeting the requirements of modern multimedia communication.

To turn information into education, it is necessary that the communication model properly meets the needs of current users.

In particular, the choice of the multimedia format becomes important or even critical.

Multimedia tools can easily capture the attention and interest of the users, thanks to their immediate interactive capacity (duration, execution mode, etc.).

In this regard, the results of a recent research funded by Microsoft are particularly interesting, as they document that the ability of young people to maintain their attention has on average decreased from 12 seconds in 2004 to today's 8 seconds (on average, a goldfish maintains its attention for about 9 seconds) [62].

Several aspects should be taken into account for the construction of this complex communicational alchemy

a) The different age of our patients

b) The different HL and eHL levels 
c) The different scopes of intervention, i.e. whether it should only be "educational" or whether it should affect the complexity of behaviors and lifestyles [63].

d) Achieving an adequate and persistent engagement of the person with diabetes. Not easy task with younger people, as they often show reduced abilities to focus and pay attention [62].

All these requirements are seemingly difficult to reconcile.

Not surprisingly, the multimedia format that, more or less, seems to embrace all these different features is actually the "commercials" format, i.e. short video advertisements [64].

In their simplicity, dynamics, their empathic capacity, their persuasive power-direct or hidden-multimedia commercial tools are able to meet the different needs related to age, HL level, purpose, and engagement.

Based on this insight, our group has been working on a new Educational Video format project, sponsored by the Associazione Medici Diabetologi Italiana (AMD)

During the XXI AMD National Congress, held in Naples in May 2017, we presented the iDIAbeLOGANDO project, where we have outlined the main features of these new video formats. The first stage of the iDIAbeLOGANDO project also included the preparation and production of these new educational video clips. Before the Covid 19 Pandemic, the deadline for the first stage of the project was scheduled for the end of 2020 [65].

\section{Conclusions}

It is clear that the approach to a state-of-the-art TE, especially in light of the ongoing health emergency, requires a change of pace, the ability to look beyond or at least to experiment with new ways. This has become a mandatory choice that can no longer be postponed.

The choice of these new approaches is not estranged from the past. It is actually a natural evolution: it is necessary in order to build an increasingly digital and changeable future.

Even the use of methods that can appear unorthodox to the scientific world, such as those of "Digital Marketing," are therefore in line with future therapeutic scenarios, in order to reach ever more patients.

\section{Acknowledgements}

Mr Giancarlo Avellino for free writing Assistance-Professor Vincenzo Casolaro MD-PHD for his supervisor's contribution.

\section{References}

1. IDF DIABETES ATLAS (2019) $9^{\text {th }}$ Edition.

2. Diabetes (2000) World Health Organization.

3. Anni 2000-2016 IL DIABETE IN ITALIA. 20 luglio 2017.

4. Branca MT, Buzzetti R, Fedele D, Giorda CB, Manicardi V, et al. (2012) PERIODICO DI APPROFONDIMENTO SCIENTIFICO, FORMAZIONE, modelli assistenziali. Rivista dell'Associazione Medici Diabetologi 15.

5. National AMD for A Predictive, Preventive, Personalized and Participative Program Diabetology (2017) XXI Congress National, Naples.

6. LA PROGRAMMAZIONE DEL FABBISOGNO DI PERSONALE MEDICO, PROIEZIONI PER IL PERIODO 2018-2025: CURVE DI PENSIONAMENTO E FABBISOGNI SPECIALISTICI.

7. Bruno G, Aimone B, Panero F (2008) Costi diretti e indiretti del diabete: un trend in continua ascesa-il Diabete 20.
8. http://www.siditalia.it/news/1751-14-11-2017-osservatorio-arnodiabete-il-profilo-assistenziale-della-popolazione-con-diabete

9. Coronavirus, cosa sapere sui bonus per le famiglie: Cittadinanzattiva aggiorna la Guida web.

10. INDICAZIONI AD INTERIM PER UN UTILIZZO RAZIONALE DELLE PROTEZIONI PER INFEZIONE DA SARS-COV-2 NELLE ATTIVITÀ SANITARIE E SOCIOSANITARIE (ASSISTENZA A SOGGETTI AFFETTI DA COVID-19) NELL'ATTUALE SCENARIO EMERGENZIALE SARS-COV-2 (2020) Rapporto ISS COVID-19 2.

11. ISS COVID-19 report no. 12/2020-Interim indications for telemedicine assistance services during the COVID-19 health emergency. April 13, 2020.

12. Emergenza COVID-19 Procedura per la realizzazione in remoto delle visite di controllo ambulatoriali programmate nei centri di diabetologia che già seguono le persone con diabete. AMD-SID-SIE.

13. COVID-19: For patients, an hour every day with AMD on Facebook. AMD.

14. Standard italiani per la cura del diabete mellito (2018) Associazione Medici Diabetologie.

15. Introduction: Standards of Medical Care in Diabetes-2019.

16. ht t ps:// w w w. idf.org/ c o m p on e nt/ attachments $/$ ?task=download\&id $=1268$

17. Miller LV, Goldstein J (1972) More efficient care of diabetic patients in a county hospital setting. New England J Med 286: 1388-1391.

18. Lacroix A, Assal J-Ph (2003) Therapeutic Education of Patients New Approaches to Chronic Disease $2^{\text {nd }}$ Edition. Maloine (eds) $2^{\text {nd }}$ Edition, Paris.

19. Assal J-Ph, Berger M, Canivet J (1982) History and aims of the diabetes education study group. International Congress Series No. 624, Excerpta Medica, Amsterdam 3-7.

20. Gillett M, Dallosso HM, Dixon S, Brennan A, Carey ME, et al. (2010) Delivering the diabetes education and self-management for ongoing and newly diagnosed (DESMOND) programme for people with newly diagnosed type 2 diabetes: cost effectiveness analysis. BMJ 341: $c 4093$.

21. He X, Li J, Wang B, Yao Q, Li L, et al. (2016) Diabetes self-management education reduces risk of all-cause mortality in type 2 diabetes patients: a systematic review and meta-analysis. Endocrine 55: 712731.

22. Heinrich E, Schaper NC, de Vries NK (2010) Self-management interventions for type 2 diabetes: a systematic review. Eur Diabetes Nurs 7: 71-76.

23. LINEE GUIDA PER LA COMUNICAZIONE ON LINE IN TEMA DI TUTELA E PROMOZIONE DELLA SALUTE (2010) Ministero della Salute.

24. Overland JE, Hoskins PL, McGill MJ, Yue DK (1993) Low literacy: a problem in diabetes education. Diabet Med 10: 847-850.

25. Rothman R, Malone R, Bryant B, Horlen C, DeWalt D, et al. (2004) The relationship between literacy and glycemic control in a diabetes disease-management program. Diabetes Educ 30: 263-273.

26. Schillinger D, Barton LR, Karter AJ, Wang F, Adler N (2006) Does literacy mediate the relationship between education and health outcomes? A study of a low-income population with diabetes. Public Health Rep 121: 245-254.

27. Porta $M$, Trento $M$, on behalf of the ROMEO Writing Committee (2004) ROMEO: Rethink organization to improve education and outcomes. Diabetic Medicine 21: 644-645. 
28. Miselli V, Monzali E, Accorsi P, Bedogni C, Pagliani U, et al. (2009) Valutazione di un intervento secondo il modello assistenziale della Group Care nel diabete di tipo 2. GIORNALE ITALIANO DI DIABETOLOGIA E METABOLISMO 29: 189-196.

29. Attività e progetti del Gruppo Italiano di Studio Educazione e Diabete (GISED) (2009) il Diabete.

30. Gentile S (2009) II progetto educazione terapeutica strutturata (ets) di AMD. II Giornale di AMD 12: 40-48.

31. Ciardullo AV, Daghio MM, Fattori G, Giudici G, Rossi L, et al. (2010) Effectiveness of the kit Conversation Map in the therapeutic education of diabetic people attending the Diabetes Unit in Carpi, Italy. Recenti Prog Med 101: 471-474.

32. Ghafoor E, Riaz M, Eichorst B, Fawwad A, Basit A (2015) Evaluation of diabetes conversation map $^{\mathrm{TM}}$ education tools for diabetes selfmanagement education. Diabetes Spectr 28: 230-235.

33. Creating the foundation for Personal Health Engagement ${ }^{\mathrm{TM}}$ and SelfManagement Education.

34. Agrusta M, Rossi A, Scarpa L, 2009 II programma educazionale strutturato Diabetes Conversation Maps nella gestione terapeutica del Diabete Mellito tipo 2. Paper presented at: Congresso AMD 2009; Rimini, Italy.

35. Srulovici E, Kay C, Rotem M, Golfenshtein N, Balicer R, et al. (2015) Diabetes Conversation Maps and health outcomes: a systematic literature review. Value Health 18: PA617-A618.

36. Trento $\mathrm{M}$, Tomelini M, Basile M, Borgo E, Passera P, et al. (2007) Il modello assistenziale della Group Care e i corsi residenziali su: management del diabete tipo 2 mediante Group Care del Dipartimento di Medicina Interna, Laboratorio di Pedagogia Clinica, Università di Torino. ATTIVITÀ DIABETOLOGICA E METABOLICA IN ITALIA.

37. Cotterez A, Durant N, Agne AA, Cherrington AL (2014) Internet Interventions to Support Lifestyle Modification for Diabetes Management: A Systematic Review of the Evidence. J Diabetes its Complications 28: 243-251.

38. Jamie Hartmann-Boyce, Mahtani KR (2020) Supporting people with long-term conditions (LTCS) during national emergencies.

39. Holtz B, Lauckner C (2012) Diabetes Management via Mobile Phones: A Systematic Review. Telemedicine and e-health 18.

40. https://www.pewresearch.org/internet/2012/11/08/mainfindings-6/ Page 1 of 15

41. Huang Z, Solijak M, Otto Behm B, Car J (2018) Clinical Relevance of Smartphone apps for diabetes management: A global Overview. Diabetes Metab Res Rev 34: e2990.

42. Whitehead L, Seaton P (2016) The Effectiveness of Self-Management Mobile Phone and Tablet Apps in Long-term Condition Management: A Systematic Review. Journal of medical Internet research 18: e97.

43. Shen Y, Wang F, Zhang X, Zhu X, Sun Q, et al. (2018) Effectiveness of Internet-Based Interventions on Glycemic Control in Patients with Type 2 Diabetes: Meta-Analysis of Randomized Controlled Trials. J Med Internet Res 20: e172.

44. Cui M, Wu X, Mao J, Wang X, Nie M (2016) T2DM Self-Management via Smartphone Applications: A Systematic Review and MetaAnalysis. PLoSONE 11: e0166718.
45. Samoocha D, Bruinvels DJ, Elbers NA, Anema JR, van der Beek AJ (2010) Effectiveness of Web-based Interventions on Patient Empowerment: A Systematic Review and Meta-analysis. J Med Internet Res 12: e23.

46. Pereira K, Phillips B, Johnson C, Vorderstrasse A (2015) Internet delivered diabetes self-management education: a review. Diabetes Technol Ther 17: 55-63.

47. Nicola M, Alsafi Z, Sohrabi C, Kerwan A, Al-Jabir A, et al. (2020) The socio-economic implications of the coronavirus pandemic (COVID-19): A review. Int J Surg 78: 185-193.

48. Mayberry LS, Lyles CR, Oldenburg B, Osborn CY, Parks M, et al. (2019) mHealth Interventions for Disadvantaged and Vulnerable People with Type 2 Diabetes. Curr Diab Rep 19: 148.

49. Scribd will begin operating the Slide Share business on December 1, 2020.

50. ISTAT data 2019.

51. Prensky M (2001) Digital Natives, Digital Immigrants. MCB University Press 9.

52. Gabarron E, Årsand E, Wynn R (2018) Social Media Use in Interventions for Diabetes: Rapid Evidence-Based Review. J Med Internet Res 20: e10303.

53. Leong AY, Sanghera R, Jhajj J, Desai N, Jammu BS, et al. (20118) Is YouTube Useful as a Source of Health Information for Adults With Type 2 Diabetes? A South Asian Perspective. Can J Diabetes 42: 395403.

54. Gimenez-Perez G, Robert-Vila N, Tomé-Guerreiro M, Castells I, Mauricio D (2018) Are YouTube videos useful for patient selfeducation in type 2 diabetes? Health Informatics J 26: 45-55.

55. Tackett S, Slinn K, Marshall T, Gaglani S, Waldman V, et al. (2018) Medical Education Videos for the World: An Analysis of Viewing Patterns for a YouTube Channel. J Association of American Medical 93: 1150-1156.

56. Hartmann-Boyce J, Morris E, Goyder C, Kinton J, Perring J, et al. (2020) Managing diabetes during the COVID-19 pandemic. CEBM The Centre for Evidence-Based Medicine develops, promotes and disseminates better evidence for healthcare.

57. Wang A, Zhao W, Xu Z, Gu J (2020) Timely blood glucose management for the outbreak of 2019 novel coronavirus disease (COVID-19) is urgently needed. Diabetes Res Clin pract 162: 108118.

58. Madathil CK, Rivera-Rodriguez AJ, Greenstein JS, Gramopadhye AK (2015) Healthcare information on YouTube: A systematic review. Health Informatics 21: 173-194.

59. Tenderich A, Tenderich B, Barton T, Richards SE (2019) What Are PWDs (People With Diabetes) Doing Online? A Netnographic Analysis. J Diabetes Sci Technol 13: 187-197.

60. Gabarron E, Dorronzoro E, Bradway M, Rivera-Romero O, Wynn R, et al. (2018) Preferences and interests of diabetes social media users regarding a health-promotion intervention. Dove press 12: 2499 2506.

61. Woo BKP (2018) What Can We Learn From Diabetes-Related YouTube Videos? Can J of Diab 45: 342

62. Technology has reduced our attention thresholds. To say it is Microsoft 
63. Abed MA, Himmel W, Vormfelde S, Koschack J (2014) Video-assisted patient education to modify behavior: A systematic review. Patient Education and Counseling 97: 16-22.

64. Kickbusch I, Pelikan JM, Apfel F, Tsouros AD (2013) Health literacyThe solid facts. World Health Organization-The Regional Office for Europe 59.

65. XXICongresso nazionale. Napoli, Maggio 2017 AMD nazionale per una diabetologia predittiva, preventiva, personalizzata e partecipativa programma. 